\title{
Gas Steady-state Diffusion in Fractal Porous Media
}

\author{
Du Zhehua $^{1}$ \\ ${ }^{1}$ Wuhan Second Ship Design and Research Institute, 430205 Wuhan, China
}

\begin{abstract}
Gas diffusion in fractal pores does not follow the classic Fick's and Knudsen's laws, so more research on gas diffusion in fractal porous media is needed. Fractal pore models are generated using the random walk method. The gas diffusion governing equations for the fractal pores are derived from the classic kineti theory of gases. The gas diffusion model is used to study the gas diffusion in fractal porous meida and to determine steady-state diffusion coefficient formulas. The results show that the diffusion coefficient is proportional to the mean proe diameter, porosity, and the exponetial function of the fractal dimension in the Knudsen diffusion regime. The diffusion coefficient is not only related to the three pore parameters but is also related to the molecular mean free path in the configurational diffusion regime.
\end{abstract}

\section{Introduction}

In the field of energy and chemical industry, there are many gas-solid reactions such as coal combustion and limestone desulfurization. Coal and limestone are typical porous media and their pore structure has fractal characteristics[1]. These porous media act as reactants or carriers. The chemical reaction between gas and porous solid is controlled by gas diffusion in the pores. Gas diffusion process in the pore has a great influence on the reaction. Classical gas-solid reaction models such as random hole model and distributed hole model are described by the traditional diffusion law (Fick's diffusion and Knudsen's diffusion). However, it is found through research that the traditional diffusion law is not applicable to gas diffusion in the fractal hole [2]. Therefore, the study of gas diffusion in fractal porous media has important engineering application value and significance.

A lot of experiments are needed to obtain the general steady-state diffusion law of fractal porous media. The experiment of gas diffusion in fractal porous media is difficult. The numerical model study has the advantage in this respect. The steady-state diffusion law of gas in fractal porous media can be obtained by a lot of numerical simulation. At present, fractal porous media diffusion models can be divided into continuous model and discrete model. Continuous model such as dust-gas model and random pore model assume that the porous medium is a continuum with uniform pore structure. However, continuous model cannot reflect the fractal characteristics of pore structure in porous media and is modified with empirical parameters, so continuous model cannot reflect the physical dispersion overpass of real material. With the development of computer, discrete model regards porous media model as composed of discrete grids and adopts Monte-Carlo square method to simulate the movement of gas molecules in the porous media model. Discrete model is more advanced than continuous model. However, there is still a large gap between pore structure of its multi-porous mesosities and that of real porous mesosities (such as coal). In addition, the simulation of gas molecular movement in the hole is a pure mathematical simulation method, which cannot reflect the free movement law of molecules in physical essence. Cao and He proposed a gas diffusion model for fractal porous media based on molecular motion theory [3].

The diffusion model proposed by $\mathrm{Cao}$ and $\mathrm{He}$ is improved in this paper. First, a random walk method is used to generate a fractal pore model of $400 \times 400 \times 400$ grids, and the pore structure is described by porosity, average pore size, and fractal dimension. Then based on the theory of molecular motion of gas, the governing equation of molecular motion is established according to four different situations of pore structure. Through a large number of numerical simulations, it is found that gas diffusion in fractal porous media is strongly affected by the pore structure. Finally, the expression of steadystate diffusion coefficient is obtained.

\section{Modeling process}

\subsection{Fractal hole model}

The random walk method proposed by Liang et al. [4] is used to generate a porous medium model with fractal characteristics. The seeds (hole grids) are randomly distributed in $400 \times 400 \times 400$ discrete cube grids (solid grids). The fractal pore models with different pore structures can be generated by adjusting the number of seeds and threshold values of random walk. Since pore structure is very complex and has fractal characteristics, it is necessary to comprehensively describe pore connectivity characteristics, pore diameter distribution,

\footnotetext{
*Corresponding author: shunli878@163.com
} 
pore volume, and pore surface area. Porosity, average pore diameter, and fractal dimension are used to describe the pore structure characteristics, which can more fully describe the above pore characteristics. The porosity $\varepsilon$ is the ratio of pore volume to the total volume of particles. The average pore diameter $d_{m}$ is 4 times the ratio of pore volume to pore area. The fractal dimension is defined in the following formula. In the formula, $D_{f}$ is fractal dimension, $r$ is the pore diameter of multi-porous medium, and $S(r)$ is the pore area corresponding to the pore size of porous medium at pore size $r$.

$$
D_{f}=-\frac{\ln (d S(r) / d r)}{\ln r}
$$

\subsection{Governing equation of gas molecule diffusion}

It is assumed that side length of a unit cube grid is $L$, the number of gas molecules in a certain grid is $N_{0}$, molecular mean free path is $\lambda$, and molecular average velocity is $v$. According to molecular motion theory, the number of $N$ molecules whose free path is greater than $x$ in $N_{0}$ molecules can be deduced.

$$
N=N_{0} e^{-\frac{x}{\lambda}}
$$

Then, the number of molecules that escape from a certain surface of cube grid in unit time without collision between molecules is the following equation.

$$
d N=\frac{N_{0}}{6 L} v\left(1-e^{-\frac{L}{\lambda}}\right)
$$

Based on equation (3), according to the connectivity of hole grid $(i, j, k)$ along the direction $i$, it can be divided into 4 situations as shown in Fig. 1 (in the figure, white is the hole grid and black is the solid grid).
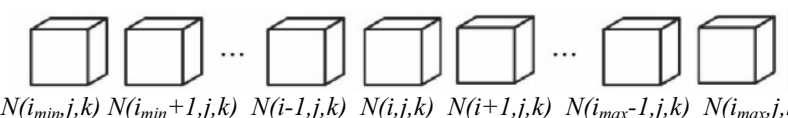

$N\left(i_{\min , j}, k\right) N\left(i_{\min }+1, j, k\right) \quad N(i-1, j, k) \quad N(i, j, k) \quad N(i+1, j, k) \quad N\left(i_{\max }-1, j, k\right) \quad N\left(i_{\max }, j, k\right)$ (a) Situtation 1
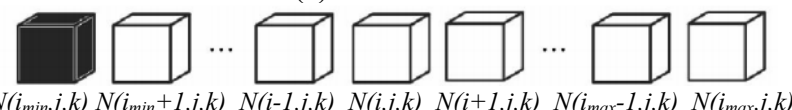

(b) Situtation 2

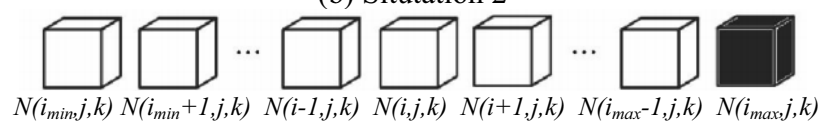

(c) Situtation 3

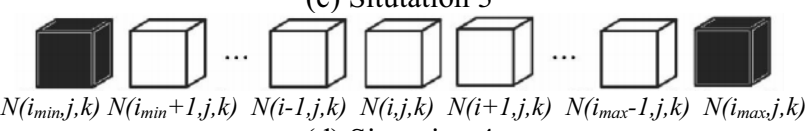

(d) Situtation 4

Fig. 1. Four situations for establishing diffusion governing equations in discrete grids

The governing equations of molecular diffusion are established respectively, and the specific process is as follows.

If $(i, j, k)$ is directly connected to external large space along direction $i$, the number of molecules added in the $(i, j, k)$ grid per unit time is as follows.

$$
\begin{aligned}
& \frac{d N(i, j, k)}{d t}=\sum_{h=i_{\min }+1}^{i_{\max }-1} \frac{N(h, j, k) v}{6 L}(1-\psi)^{2} \psi^{|h-i|-1} \\
& +\frac{N\left(i_{\min }, j, k\right) v}{6 L}(1-\psi) \psi^{i-i_{\min }-1} \\
& +\frac{N\left(i_{\max }, j, k\right) v}{6 L}(1-\psi) \psi^{i_{\max }-i-1} \\
& -\frac{N(i, j, k) v}{6 L}(1-\psi)\left(2+\frac{(1-\psi)}{\psi}\right)
\end{aligned}
$$

If $(i, j, k)$ is directly connected to the outside large space along the right side of direction $i$, while the left side is connected to a solid grid, the number of molecules increased in the grid of $(i, j, k)$ in unit time is shown in the following equation.

$$
\begin{aligned}
& \frac{d N(i, j, k)}{d t}=\sum_{h=i_{\min }+1}^{i_{\max }-1} \frac{N(h, j, k) v}{6 L}(1-\psi)^{2}\left(\psi^{|h-i|-1}+\psi^{i+h-2 i_{\min }-2}\right) \\
& +\frac{N\left(i_{\max }, j, k\right) v}{6 L}(1-\psi)\left(\psi^{i_{\max }-i-1}+\psi^{i_{\max }+i-2 i_{\min }-2}\right) \\
& -\frac{N(i, j, k) v}{6 L}(1-\psi)\left(2+\frac{(1-\psi)}{\psi}\right)
\end{aligned}
$$

If $(i, j, k)$ is directly connected to the outside large space along the left side of direction $i$, and the right side is connected to the solid grid, the number of molecules increased in the grid $(i, j, k)$ in unit time is shown in the following equation.

$$
\begin{aligned}
& \frac{d N(i, j, k)}{d t}=\sum_{h=i_{\min }+1}^{i_{\max }-1} \frac{N(h, j, k) v}{6 L}(1-\psi)^{2}\left(\psi^{|h-i|-1}+\psi^{2 i_{\max }-2-i-h}\right) \\
& +\frac{N\left(i_{\max }, j, k\right) v}{6 L}(1-\psi)\left(\psi^{i-i_{\min }-1}+\psi^{2 i_{\max }-2-i-i_{\min }}\right) \\
& -\frac{N(i, j, k) v}{6 L}(1-\psi)\left(2+\frac{(1-\psi)}{\psi}\right)
\end{aligned}
$$

If $(i, j, k)$ is connected to the solid grid along the left and right sides of the direction $i$, the number of molecules increased in the grid of $(i, j, k)$ in unit time is shown in the following equation.

$$
\begin{aligned}
& \frac{d N(i, j, k)}{d t}=\sum_{h=i_{\min }+1}^{i_{\max }-1} \frac{N(h, j, k) v}{6 L}(1-\psi)^{2} \psi^{|h-i|-1} \\
& +\frac{N(i, j, k) v}{6 L}(1-\psi)\left(2+\frac{(1-\psi)}{\psi}\right)+\frac{(1-\psi)^{2}}{1-\psi^{2\left(i_{\max }-i_{\min }-1\right)}} \\
& \sum_{h=i_{\min }+1}^{i_{\max }-1} \frac{N(i, j, k) v}{6 L}\left(\psi^{2 i_{\max }-h+i-2 i_{\min }-3}+\psi^{2 i_{\max }-i+h-2 i_{\min }-3}\right. \\
& \left.+\psi^{i+h-2 i_{\min }-2}+\psi^{2 i_{\max }-2-i-h}\right)
\end{aligned}
$$

The above is diffusion governing equation in the $i$ direction. Similarly, the diffusion governing equations in the $j$ direction and $k$ direction can be derived. The governing equation of gas molecular diffusion is established from the basic physical principles according to the specific situation of pore structure, so the diffusion model can reflect the diffusion mechanism. 


\section{Calculation results and discussion}

The diffusion model in this paper is used to simulate gas diffusion in 10 groups of fractal hole models under different diffusion gas and diffusion temperature conditions. The simulated boundary conditions are as follows: one end of gas molar concentration is 41.6 $\mathrm{mol} / \mathrm{m}^{3}$, the other end is $20.8 \mathrm{~mol} / \mathrm{m}^{3}$. The pore structure parameters of 10-component shaped hole model with unit mesh size $L=1 \mathrm{~nm}$ are listed in Table 1 .

Table 1. Pore structure parameters of fractal hole model $(L=$ $1 \mathrm{~nm})$

\begin{tabular}{|c|c|c|c|}
\hline Serial number & $\boldsymbol{\varepsilon} / \%$ & $\boldsymbol{d}_{\boldsymbol{m}} / \mathbf{n m}$ & $\boldsymbol{D}_{\boldsymbol{f}}$ \\
\hline Group 1 & 31.41 & 7.96 & 2.42 \\
\hline Group 2 & 34.43 & 8.36 & 2.01 \\
\hline Group 3 & 40.09 & 6.99 & 2.15 \\
\hline Group 4 & 46.36 & 7.07 & 2.13 \\
\hline Group 5 & 47.38 & 7.22 & 1.99 \\
\hline Group 6 & 52.57 & 6.91 & 1.85 \\
\hline Group 7 & 57.11 & 7.05 & 1.64 \\
\hline Group 8 & 60.28 & 6.75 & 1.71 \\
\hline Group 9 & 62.04 & 6.04 & 1.60 \\
\hline Group 10 & 68.44 & 5.85 & 1.48 \\
\hline
\end{tabular}

Gas diffusion in porous media can be divided into Knudsen diffusion region, main diffusion region and transition region. Gas diffusion of porous media commonly used in engineering mainly occurs in Knudsen diffusion zone and transition zone, so this paper only studies diffusion coefficients of these two zones. Since three parameters are used to comprehensively describe pore structure and its influence on pore diffusion, it is difficult to intuitively study combined effects of three parameters. To solve this problem, the influence of average pore size on diffusion coefficient can be studied under the condition of constant porosity and fractal dimension by increasing grid size.

\subsection{Influence of diffusion gas and temperature}

The mean free path $\lambda$ of simulated diffusion molecules is about $90 \mathrm{~nm}$. Then for the 10 groups of hole models shown in Table 1 , when $L=2 \mathrm{~nm}$ and $L=50 \mathrm{~nm}$, the diffusion can be considered to be in Knudsen zone and transition zone, respectively. The diffusion of hydrogen, helium, neon, nitrogen, oxygen, and carbon dioxide in $L=2 \mathrm{~nm}$ and $L=50 \mathrm{~nm}$ pore models at $293 \mathrm{~K}$ and nitrogen at $293 \mathrm{~K}, 343 \mathrm{~K}, 393 \mathrm{~K}, 433 \mathrm{~K}, 493 \mathrm{~K}$ are calculated. Figure 2 shows the relationship between diffusion coefficient of Group10 hole model at $L=2 \mathrm{~nm}$ and $L=50 \mathrm{~nm}$, temperature and molar mass of diffusion gas. The result shows that the relationship between diffusion coefficient and molar mass and temperature of diffused gas is as follows.

$$
D \propto \sqrt{T / M}
$$

In the formula, $D$ is steady-state diffusion coefficient, $T$ is diffusion temperature, and $M$ is molar mass of diffuse gas. In Knudsen zone and transition zone, diffusion coefficient is proportional to average molecular velocity, which is consistent with theoretical analysis and existing experimental results [5].

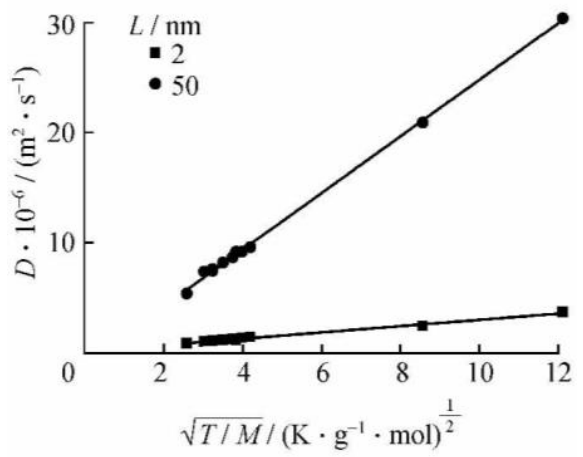

Fig. 2 Influence of diffusion gas and temperature on diffusion coefficient (Group 10)

\subsection{Influence of pore structure}

Due to the different diffusion mechanism in different diffusion zones, the influence of pore structure on diffusion coefficient is different, so it is necessary to study the influence of pore structure on diffusion coefficient in sections. In Knudsen diffusion region, diffusion coefficient is proportional to the average aperture. In this paper, when $L<5 \mathrm{~nm}$ or the ratio of average pore diameter to molecular average free path $D M / \lambda<0.5$, diffusion coefficient in the pore is proportional to the average pore diameter, which can be approximately considered as the diffusion in Knudsen region. In this paper, nitrogen diffusion process of the above 10 groups of porous models at $293 \mathrm{~K}$ is simulated, and diffusion coefficients of the porous models at $L=$ $1 \mathrm{~nm}-3 \mathrm{~nm}$ are calculated. Figure 3 shows the relationship between diffusion coefficient of Knudsen area and the average pore diameter and porosity. The result shows that diffusion coefficient is proportional to the product of the average pore size and porosity. Diffusion coefficient formula of Knudsen region can be obtained by fitting calculated data.

$$
D=\frac{v}{2} d_{m} \varepsilon e^{3.39-2.42 D_{f}}
$$

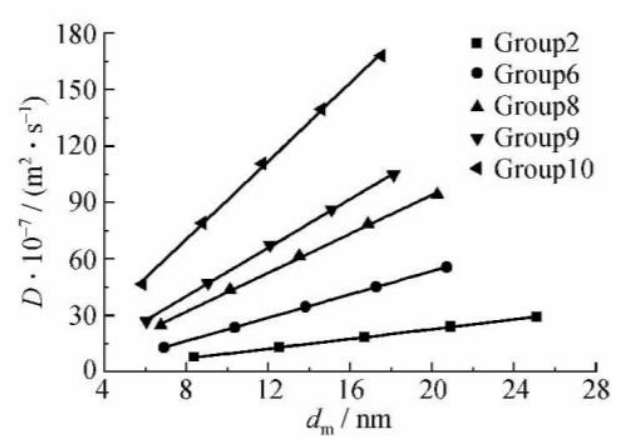

Fig. 3 Influence of average pore size and porosity on diffusion coefficient in Knudsen region 
When $0.5<d_{m} / \lambda<10$, diffusion is in the transition zone. Similarly, diffusion coefficient formula of transition zone can be calculated by simulating the diffusion of pore model with $L=5 \mathrm{~nm} \sim 100 \mathrm{~nm}$. Figure 4 shows the relationship between diffusion coefficient of transition zone and the average pore size, which can be approximated by an exponential function.

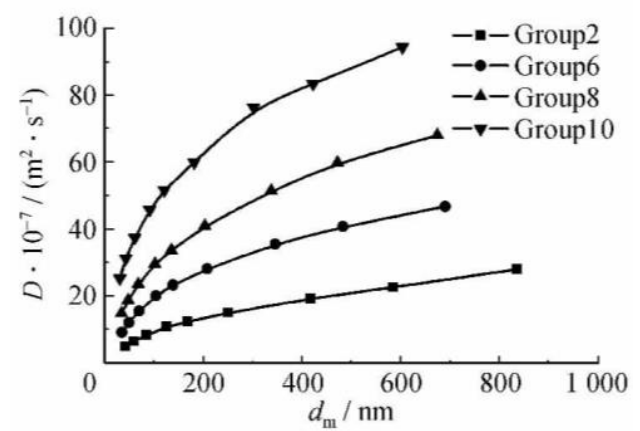

Fig. 4 Influence of average pore size on diffusion coefficient in transition zone

\section{Conclusion}

Gas diffusion coefficient is proportional to the average velocity of gas molecules.

In Knudsen diffusion region, the diffusion is obviously affected by pore structure. Under the same fractal dimension, diffusion coefficient is proportional to the product of mean pore size and porosity, but independent of the mean free path of molecule.

In transit region, diffusion coefficient is not only related to pore structure parameters, but also related to molecular mean free path. Under different ratio of average pore diameter to average molecular free path, pore structure has different effect on the diffusion rate.

\section{Acknowledgments}

This work was supported by the National Key Research and Development Program (No.2017YFC0307800) .

\section{References}

1. A.K. Katz, A.O. Thompson, Physical Review Letters, 54, 3558 (1985)

2. M. Costa, A. Araujo, Physical Review E, 67, 1296 (2003)

3. L. Cao, R. He, Combust Sci. and Tech., 182, 822 (2010)

4. Z. Liang, R. He, Combust Sci. and Tech., 179, 637 (2007)

5. R. He, X.C. Xu, C.H. Chen, Fuel, 77, 1291 (1998) 